\title{
Congenital Fibular Sesamoid Aplasia: A case report
}

\author{
by Tugrul Alici, $\mathbf{M D}^{1} \rrbracket$, Semih Dedeoglu, $\mathbf{M D}^{2}$, Yunus Imren, $\mathbf{M D}^{3}$, Hakan Gundes, $\mathbf{M D}^{4}$
}

The Foot \& Ankle Journal 2 (2): 1

Congenital absence of the lateral sesamoid bone is a relatively rare condition. Literature review reveals very few case presentations relevant to this condition. We present a case of lateral sesamoid aplasia that was incidentally detected upon roentgenograms of a patient presenting with a fracture to the base of the proximal phalanx.

Key words: Lateral sesamoid, fibular sesamoid, phalangeal fracture, aplasia, absent sesamoid

Accepted: January, $2009 \quad$ Published: February, 2009

This is an Open Access article distributed under the terms of the Creative Commons Attribution License. It permits unrestricted use, distribution, and reproduction in any medium, provided the original work is properly cited. (The Foot \& Ankle Journal (www.faoj.org)

\section{Case Report}

A 31 year-old male hit his right foot during a rafting event. The patient presents with pain and swelling to the right foot. Previous treatment included cold compression and irregular use of analgesics for pain and swelling. The physical examination demonstrated edema around the first metatarsophalangeal joint, palpation-induced tenderness at the medial aspect of the joint, with complete and painful range of motion. Further examination with plane foot anteroposterior radiographs showed a medial, non-displaced fracture at the base of proximal phalanx. (Fig.1)

\footnotetext{
Address correspondence to: Tugral Alici, MD

Department of Orthopedics and Traumatology

University of Maltepe, Istanbul, Turkey

Feyzullah Cad. No:39 34843

Email: tugrulalici71@hotmail.com

${ }^{1}$ Asistant Prof. , Department of Orthopaedics \& Traumatology, Maltepe University, Istanbul.

${ }^{2}$ Department of Orthopaedics \& Traumatology, Vakif Gureba Training and Research Hospital, Istanbul.

${ }^{3}$ Department of Orthopaedics \& Traumatology, Vakif Gureba Training and Research Hospital, Istanbul.

${ }^{4}$ Prof., Department of Orthopaedics \& Traumatology, Maltepe

University, Istanbul.
}

In addition, the lateral or fibular sesamoid appeared aplasic or absent in routinely-ordered foot axial sesamoid radiograph. (Fig.2) The patient was questioned about any previous problems relating to his right foot. The patients other sport activity included basketball once or twice a week. He denies any injury to his right foot from this activity and has had no previous foot surgery. Physical examination of the right foot was considered as normal except of the signs associated with the proximal phalangeal fracture. It was decided that this variation had no bearing on functional loss or activity for the patient. He was placed in a short leg circular cast, non-weight bearing for 2 weeks. The cast was then removed and partial weight bearing was provided through soft shoes. Complete weight bearing was allowed at 4 weeks and sport activities was permitted at the end of 2 months. A 6-month follow-up of patient demonstrated that the lateral or fibular sesamoid aplasia, considering his younger age, did not adversely influence his attendance to sport activities before or after injury. 


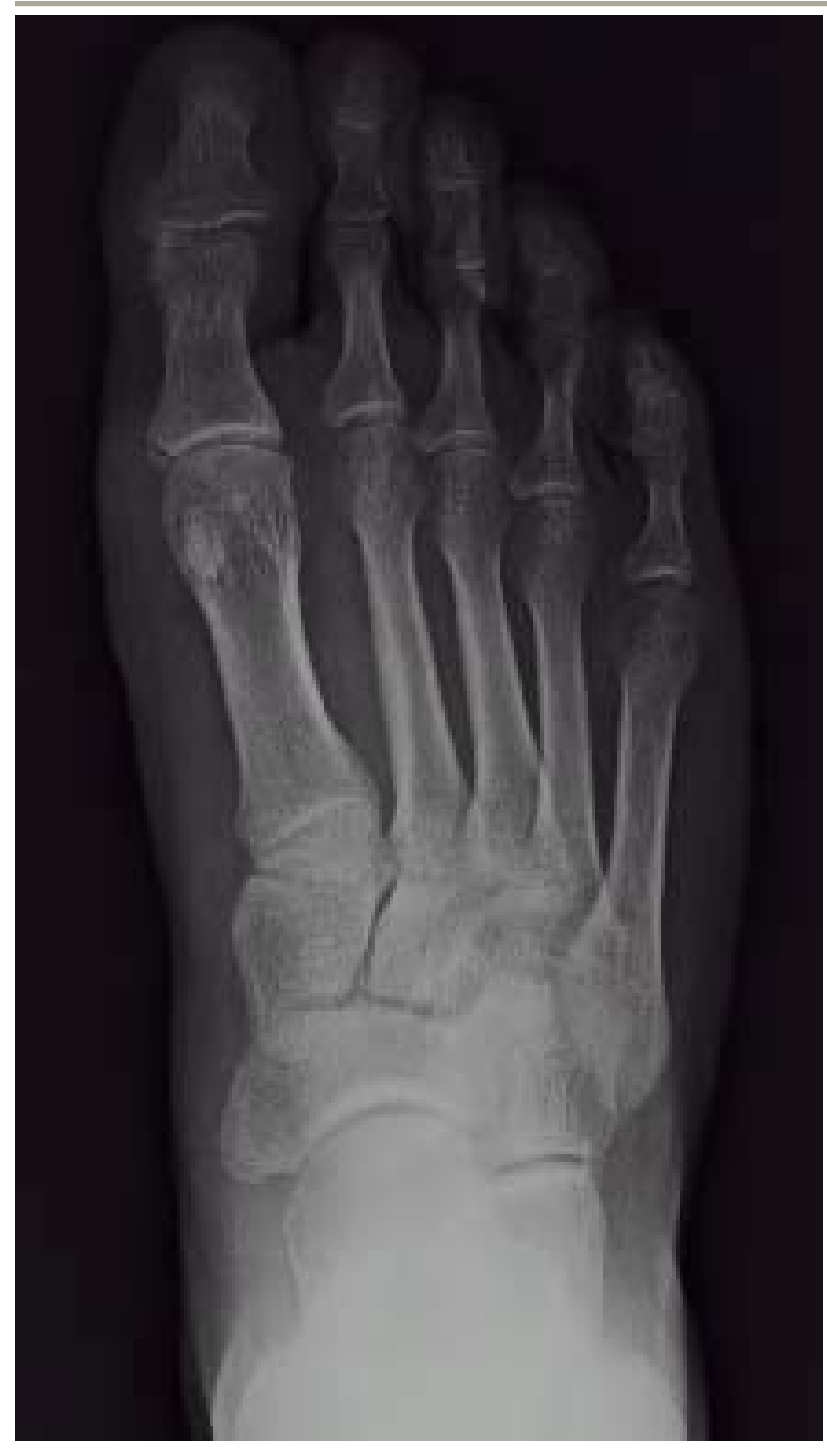

Figure 1 Radiograph showing medial, nondisplaced, proximal phalangeal fracture. Aplasia or an absent fibular sesamoid is also seen.

\section{Discussion}

Sesamoid bones of the foot originate from a cartilage bud at the $12^{\text {th }}$ gestational week. ${ }^{1}$ Ossification usually occurs between 8 and 10 years of age. ${ }^{5}$ Inferior contact surfaces of metatarsal heads become flattened with compression and form the intersesamoid ridge

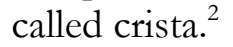

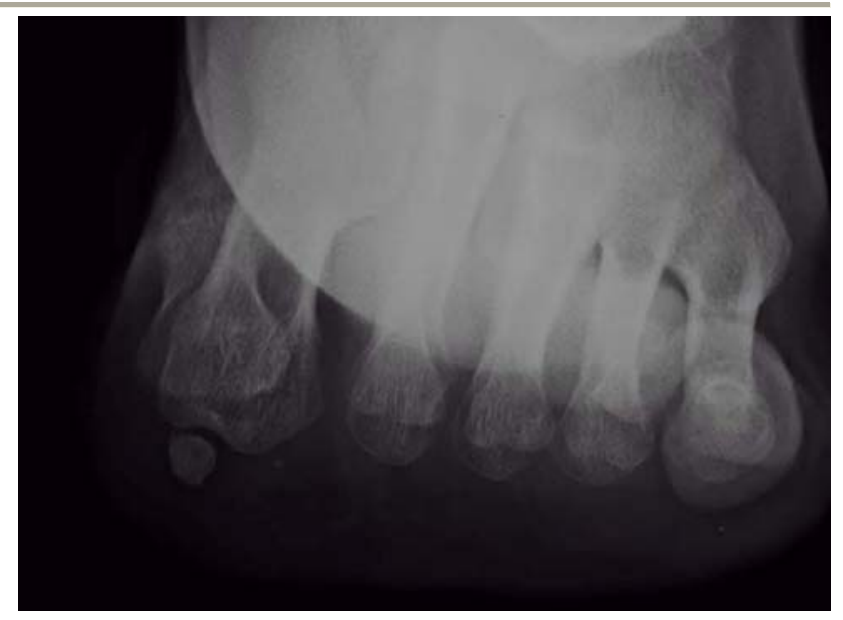

Figure 2 Axial sesasmoid radiograph reveals absent fibular sesamoid with a slightly visable ossified region central to the aplastic sesasmoid.

The reasons for development of sesamoid aplasia are not fully understood but it is thought to be congenital. Congenital absence or aplasia of one or two of the sesamoid bones of toe is reported to be rare. It is reported that lateral or fibular sesamoid aplasia is rarer than medial or tibial sesamoid aplasia. ${ }^{6,7}$ It is known that sesamoid bone excision in hallux valgus surgery (i.e. McBride bunionectomy) may result in varus, valgus, and hallux extensus or cock-up hallux deformities by altering the biomechanics of the toe. ${ }^{2,4}$

Similar to the reviewed literature, findings of physical examination of this aplasia was assessed to be within normal limits without rendering loss of function. The hallucal sesamoids, although small and seemingly insignificant, play an important role in the function of the great toe by absorbing weight-bearing stress, reducing friction, and protecting tendons. ${ }^{8,9}$ They are also known to exert biomechanical features similar to that of the patella by increasing the efficiency of flexor hallucis brevis muscle by elevating its lever arm. ${ }^{4,5}$ Secondary causes of aplasia may include infection. A case of sesamoid bone resorption secondary to infection was reported by Conway, et al. ${ }^{3}$ The reason for normal biomechanics of toe in congenital sesamoid aplasia may be the presence of a cartilaginous sesamoid, which is noncalcified, and hence not seen in direct roentgenogram. ${ }^{7}$ 


\section{References}

1. Brenner E, Gruber H, Fritsch H. Fetal Development of the first metatarsophalangeal joint complex with special reference to the intersesamoidal ridge. Ann Anat 184:481487, 2002.

2. Brenner E. The intersesamoidal ridge of the first metatarsal bone: anatomical basics and clinical considerations. Surg Radiol Anat. May;25(2):127-131, 2003.

3. Conway WF, Hayes CW, Murphy WA. Total resorption of the lateral sesamoid secondary to Pseudomonas aeruginosa osteomyelitis. Skeletal Radiol 18:483-484,1989.

4. Coughlin MJ. Sesamoids and accessory bones of the foot. In Mann RA, Coughlin MJ (eds) Surgery of the Foot and Ankle, 7th Edition. Mosby, St Louis, vol. 1, pp.437-499, 1999.

5. Downey MS, Merritt SC, Sharrock-Maher CJ, Bernbach MR. Digital and Sesamoid Fractures.

McGlamry's Forefoot Surgery ,LWW, Philadelphia 559573, 2004.

6. Goez J, De Lauro T. Congenital absence of the tibial sesamoid. J Am Podiatr Med Assoc 85:509-510,1995.

7. J.M. Le Minor. Congenital absence of the lateral metatarso-phalengeal sesamoid bone of the human hallux. Surg Radiol Anat. 21(3):225-227,1999.

8. Richardson EG: Hallucal sesamoid pain: causes and surgical treatment. J Am Acad Orthop Surg 7(4):270278,1999.

9. Cardona,T., Kline, A. Surgical excision of painful fibular sesamoid. The Foot and Ankle Journal 1(8):2, 2008 . 